\title{
Tecnologias desenvolvidas no contexto da saúde da mulher no Brasil: uma revisão integrativa
}

Developing Technologies in Women's Health in Brazil: An Integrated Review

Revisión integrativa de las tecnologías desarrolladas en el contexto de la salud de la mujer en Brasil

Como citar este artigo: Barros, Francisco Railson Bispo de; Lima, Raquel Faria da Silva; Magalhães, Vitória Mariana de Paula. Tecnologias desenvolvidas no contexto da saúde da mulher no Brasil: uma revisão integrativa. Revista Cuidarte. 2021;12(1):e1159. http://dx.doi.org/10.15649/cuidarte.1159

Revista Cuidarte

doi Rev Cuid. Ene. - Abril. 2021; 12(1): e1159 http://dx.doi.org/10.15649/cuidarte.1159

E-ISSN: 2346-3414

(1) Francisco Railson Bispo de Barros

(1) Raquel Faria da Silva Lima²

(1) Vitória Mariana de Paula Magalhães ${ }^{3}$

1 Centro Universitário do Norte UNINORTE, Manaus-AM/Brasil. E-mail: enf.franciscobarros@gmail.com

2 Universidade Federal do Amazonas UFAM, Manaus-AM/Brasil.

E-mail: lima.raquelfs@gmail.com

3 Centro Universitário do Norte UNINORTE, Manaus-AM/Brasil. E-mail:vitoriamariana.sz@hotmail.com

\section{Resumo}

Introdução: as tecnologias em saúde dizem respeito a tudo o que é utilizado como instrumento para cuidar e educar a outras pessoas. Diante desse conceito, esta revisão tem por objetivo conhecer as publicações científicas acerca do desenvolvimento de tecnologias voltadas para a área de saúde da mulher no Brasil. Materiais e métodos: trata-se de uma revisão integrativa, de natureza descritiva, realizada por meio de consulta as bases de dados Banco de Dados em Enfermagem (BDENF), LatinoAmericano e do Caribe de Informação em Ciências da Saúde (LILACS) e Scientific Electronic Library Online (SCIELO), no período de 2009 a 2019. Resultados: os cruzamentos de busca nas bases de dados estabelecidas resultaram na identificação de 46 estudos no banco de dados BDENF, 280 no LILACS e 55 na SciELO, totalizando 381 estudos. A aplicação dos filtros possibilitou refinamento que resultou em seleção final de 23 estudos. Discussão: a análise dos resultados evidenciou que o foco mais referido foi o desenvolvimento de tecnologias leve-duras. Quando analisada a abordagem dessas tecnologias, observouse que as temáticas mais beneficiadas pelo desenvolvimento das tecnologias em saúde foram a educação em saúde da mulher na gestação e na amamentação. Conclusões: percebe-se, por meio da revisão, que se faz necessário o desenvolvimento, validação e implementação de todas as classes das tecnologias em saúde, pois a qualidade técnica e conceitual passará por análise e testagem dos profissionais que compreendem as factuais necessidades do público foco.

Palavras chave: Tecnologia; Cuidados em saúde; Saúde da Mulher.

Recebido: 17 de marco de 2020

Aceito: 16 de septembro de 2020

Publicado: 12 de fevereiro de 2021 $\square *$ Correspondência

Francisco Railson Bispo de Barros

E-mail:enf.franciscobarros@gmail.com 


\section{Developing Technologies in Women's Health in Brazil: An Integrated Review}

\section{Abstract}

Introduction: Health technologies refer to those tools that are used to care for and educate people. Given this concept, this review aims to identify scientific publications related to developing technologies for women's health in Brazil. Materials and Methods: An integrative descriptive review was conducted through database consultations in the Brazilian Nursing Database (BDENF), Latin American \& Caribbean Health Sciences Literature (LILACS) and Scientific Electronic Library Online (SciELO) in 2009-2019. Results: 46 studies were identified in the BDENF database, 280 in LILACS and 55 in SciELO, for a total of 381 studies retrieved by using crossreferences in these databases. The final selection was refined to 23 studies by applying search filters. Discussion: The results analysis showed that the most cited topic was the development of soft-hard technologies. In analyzing the approach of these technologies, it was found that one of the topics that greatly benefited from the development of health technologies was the education in women's health during pregnancy and breastfeeding. Conclusion: This review indicated the need to continue developing, validating and applying these technologies as their technical and conceptual quality will be analyzed and verified by professionals who understand the actual needs of the target audience.

Key words: Technology; Health care; Women's Health.

\section{Revisión integrativa de las tecnologías desarrolladas en el contexto de la salud de la mujer en Brasil}

\section{Resumen}

Introducción: Las tecnologías de la salud se refieren a todas aquellas herramientas que se utilizan para cuidar y educar a los demás. Frente a este concepto, esta revisión tiene como objetivo conocer las publicaciones científicas relacionadas con el desarrollo de las tecnologías para la salud de la mujer en Brasil. Materiales y métodos: Se realizó una revisión integrativa de tipo descriptivo mediante consulta en bases de datos como el Banco de Datos de Enfermería (BDENF), Literatura Latinoamericana y del Caribe en Ciencias de la Salud (LILACS) y Scientific Electronic Library Online (SciELO) entre 2009 y 2019. Resultados: A través de referencias cruzadas en las bases de datos mencionadas, se identificaron 46 estudios en el banco de datos BDENF, 280 en LILACS y 55 en SciELO, para un total de 381 estudios. Así mismo, se refinó la selección final a 23 estudios mediante la aplicación de filtros. Discusión: En el análisis de resultados se evidenció que el tema más referenciado fue el desarrollo de tecnologías blandas-duras. Al analizar el enfoque de estas tecnologías, se observó que uno de los temas más beneficiados del desarrollo de las tecnologías de la salud fue el de la educación en materia de salud de la mujer durante el embarazo y la lactancia. Conclusiones: Con esta revisión se demostró que es necesario seguir desarrollando, validando y aplicando todas las clases de tecnologías sanitarias, cuya calidad técnica y conceptual será analizada y verificada por profesionales que comprenden las necesidades reales del público objetivo

Palabras clave: Tecnología; Cuidado de la salud; Salud de la mujer. 


\section{Introdução}

O manuseio da tecnologia se faz presente deste os primórdios da humanidade até os dias atuais, acompanhando as civilizações a datar do instante em que passaram a idealizar técnicas que facilitassem suas tarefas de vida diária'. Entre os diferentes avanços que vêm acontecendo no mundo, pode-se citar o avanço das tecnologias como processo que tem contribuído para auxiliar as ações diárias das pessoas. As mudanças também ocorrem no campo da saúde, em que se podem utilizar as chamadas Tecnologias em Saúde na assistência, educação e ensino². A expressão Tecnologias em Saúde, numa percepção mais genérica, engloba uma coleção de técnicas, protocolos, sistemas e dispositivos que são implementados no contexto da saúde para auxiliar os profissionais na prestação dos cuidados nas vertentes da prevenção, promoção e reabilitação dos mais diversos grupos da sociedade 3 .

As Tecnologias em Saúde podem ser classificadas como leve, leve-dura e dura. Essa classificação trata a tecnologia de forma abrangente, por intermédio da análise de todo o processo construtivo, até o produto final. As leves são os processos de produção de conversação, de elos, dos relacionamentos que direcionam a convergência do usuário com necessidades de ações de saúde. As leve-duras incluem os conhecimentos estruturados e caracterizados pelos princípios que operam em saúde, a exemplo da clínica médica, epidemiologia etc. Já as duras são representadas pelo produto efetivo como equipamentos, mobiliário tipo permanente ou de consumo ${ }^{4}$.

Nesse contexto, busca-se cada vez mais novas tecnologias em saúde que sejam eficazes e menos invasivas para prevenção de agravos e promoção da saúde, principalmente no cenário em que aproximadamente 830 mulheres morrem todos os dias por complicações evitáveis relacionadas à gestação, parto e puerpério no mundo, sendo que $99 \%$ ocorrem em países emergentes. Dessa forma, entende-se por novas tecnologias o conhecimento abstrato e prático, ou seja, a tecnologia é o produto da junção entre conhecimento prático e conhecimento teórico ${ }^{5-6}$.

A utilização de tecnologias na área de saúde está em avançada ascensão, visto que podem proporcionar aos profissionais atingirem maior acerto e desenvoltura em seus trabalhos, e as mulheres maior conhecimento e autonomia do autocuidado, uma vez que a política pública na área de saúde da mulher guia-se pelo

A utilização de tecnologias na área de saúde está em avançada ascensão, visto que podem proporcionar aos profissionais atingirem maior acerto e desenvoltura em seus trabalhos. paradigma humanístico. No que diz respeito a enfermagem, acredita-se que o uso das tecnologias tem modificado o modo de lidar com a assistência e recursos utilizados de forma rápida e organizada, qualificando o atendimento e fundamentando a ciência da profissão.

Nessa conjuntura, o presente estudo tem como objetivo conhecer as publicações científicas acerca do desenvolvimento de tecnologias voltadas para a área de saúde da mulher no Brasil. Para tanto, elaborou-se a seguinte indagação norteadora: "o que foi produzido em território brasileiro quanto a estudos científicos no contexto da mulher relacionado à tecnologia em saúde?" 


\section{Materiais e métodos}

A pesquisa consiste em uma revisão integrativa da literatura, tendo em vista identificar e compreender as publicações existentes na comunidade científica nacional sobre a produção de tecnologias voltadas para a saúde da mulher de janeiro de 2009 a janeiro de 2019.

Este método se configura como uma síntese do estado da arte dos dados científicos relacionados a um problema de pesquisa, possibilitando conclusões amplas a respeito de um tema específico, apontando lacunas que podem ser preenchidas por novas pesquisas ${ }^{8}$. Vale salientar que essa modalidade de pesquisa não necessita de apreciação pelo Comitê de Ética em Pesquisa (CEP), fato este respaldado pelas Resoluções 466/12 e 510/16 do Ministério da Saúde9 .

A presente revisão foi desenvolvida se embasando no processo de elaboração de revisão proposto por Souza, Silva e Carvalho, que abrange seis fases: I - Elaboração da pergunta norteadora; II - Busca ou amostragem na literatura; III - Coleta de dados; IV - Análise crítica dos estudos incluídos; V - Discussão dos resultados; VI - Apresentação da revisão integrativa ${ }^{10}$.

A questão que norteou esta revisão integrativa foi a seguinte: "o que foi produzido em território brasileiro quanto a estudos científicos no contexto de saúde da mulher relacionado à tecnologia?" Para a busca ou amostragem dos artigos foram utilizadas as seguintes bases de dados: Banco de Dados em Enfermagem (BDENF), Latino-Americano e do Caribe de Informação em Ciências da Saúde (LILACS) e Scientific Electronic Library Online (SciELO). Os estudos duplicados entre as bases de dados foram examinados uma única vez.

$\mathrm{Na}$ fase de coleta dos estudos foram utilizados os presentes critérios: estudos disponíveis na íntegra online, nos idiomas português, inglês e espanhol, estudos desenvolvidos por pesquisadores brasileiros e publicados no período compreendido entre 2009 e 2019. Para a exclusão foram considerados os seguintes critérios: estudos em que não foi possível identificar relação com a temática por meio da leitura de título e resumo e estudos secundários. Os descritores utilizados foram: tecnologia and saúde da mulher. O procedimento de seleção dos estudos está representado na Figura 1.

Para organização e tabulação dos dados dos estudos selecionados, foi utilizado um protocolo de revisão integrativa para esse fim. Nesse protocolo se fez necessário identificar o título do estudo, sua instituição-sede, o local em que o trabalho foi publicado, as características metodológicas da pesquisa e a avaliação do rigor metodológico ${ }^{10}$.

No que diz respeito a classificação do nível de evidência dos estudos fora empregado os níveis de evidência científica da Agency for Healthcare Research and Quality (AHRQ), que se compreende seis níveis: I - metanálise e revisão sistemática; II - ensaios clínicos com randomização; III - ensaios clínicos sem randomização; IV - coorte e de caso-controle; V - revisão sistemática de pesquisas descritivas e qualitativas; $\mathrm{VI}$ - estudos descritivos ou qualitativos ${ }^{11}$. 


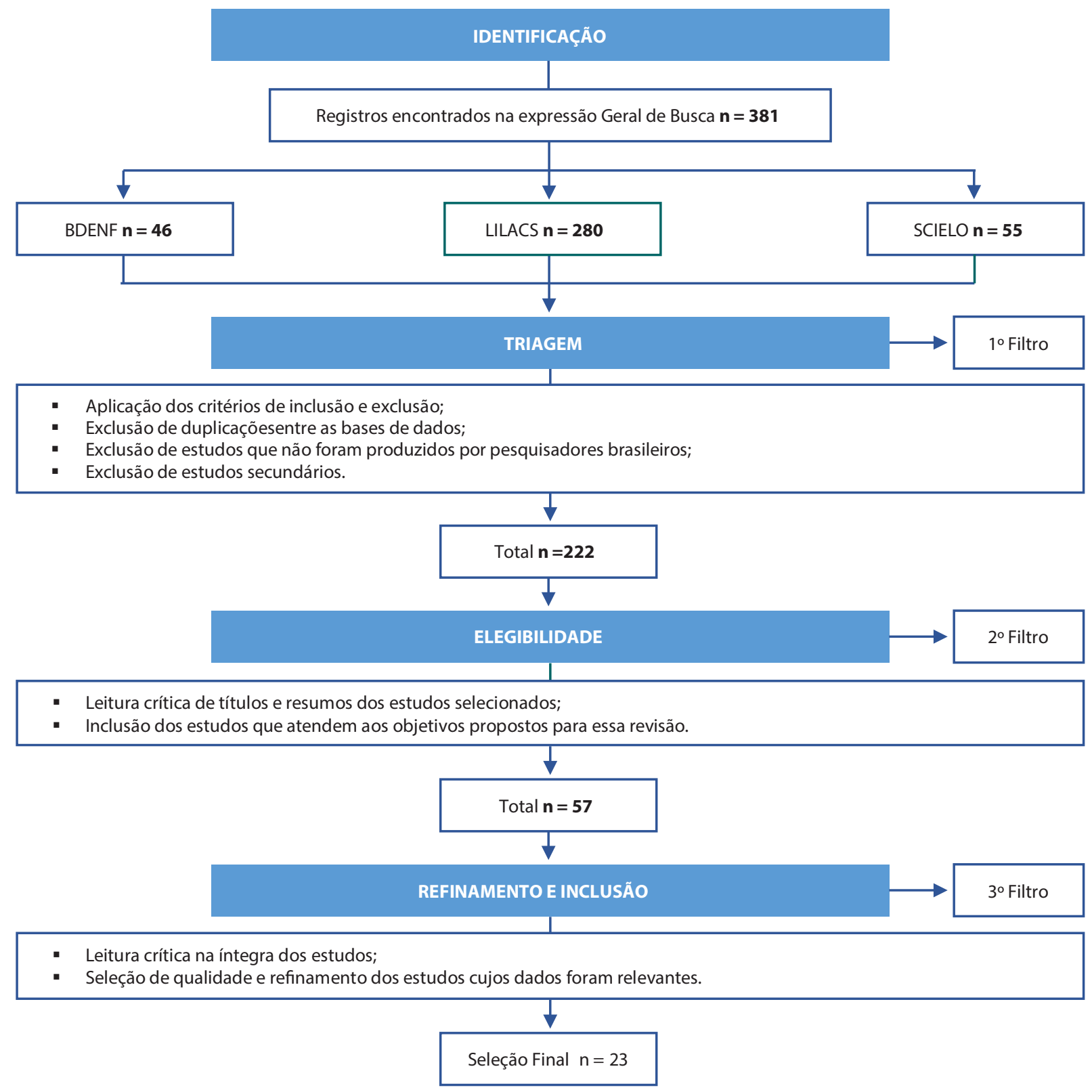

Figura 1 - Fluxograma do procedimento de seleção dos estudos. Brasil, 2020. Fonte: Autoria própria, 2020.

\section{Resultados}

Os cruzamentos de pesquisa nas bases de dados definidas resultaram na identificação de 46 estudos no banco de dados BDENF, 280 no LILACS e 55 na SciELO, totalizando 381 estudos. A aplicação dos três filtros possibilitou refinamento que resultou em seleção final

A aplicação dos três filtros possibilitou refinamento que resultou em seleção final de 23 estudos, com base na relevância e na qualidade dos dados. de 23 estudos, com base na relevância e na qualidade dos dados para a presente revisão. Para melhor identificação de cada estudo, seguiu-se uma classificação alfanumérica iniciada em E1 e finalizada em E23 (Tabela 1). 
Tabela 1 - Estudos selecionados para análise. Brasil, 2020.

\begin{tabular}{|c|c|c|c|c|}
\hline No & Ano/Autor & Título & Temática & $\begin{array}{l}\text { Tecnologia } \\
\text { utilizada }\end{array}$ \\
\hline $\mathrm{E} 1^{12}$ & $\begin{array}{l}2010 \\
\text { Nunes JM. }\end{array}$ & $\begin{array}{c}\text { Tecnologia educativa: uma proposta para a } \\
\text { promoção da saúde de um grupo de } \\
\text { mulheres }\end{array}$ & $\begin{array}{l}\text { Promoção da } \\
\text { saúde da mulher }\end{array}$ & $\begin{array}{l}\text { Atividades } \\
\text { educativas }\end{array}$ \\
\hline $\mathrm{E} 2^{13}$ & $\begin{array}{l}2010 \\
\text { Nascimento } \\
\text { NM, et al. }\end{array}$ & $\begin{array}{c}\text { Tecnologias não invasivas de cuidado no } \\
\text { parto realizadas por enfermeiras: a } \\
\text { percepção de mulheres }\end{array}$ & $\begin{array}{l}\text { Promoção do } \\
\text { conforto da } \\
\text { parturiente }\end{array}$ & $\begin{array}{l}\text { Acolhimento e } \\
\text { ações } \\
\text { humanizadas }\end{array}$ \\
\hline $\mathrm{E} 3^{14}$ & $\begin{array}{l}2011 \\
\text { Morais } \\
\text { MLC. }\end{array}$ & $\begin{array}{l}\text { Construção e validação de hipermídia } \\
\text { educacional em saúde sexual -uma } \\
\text { abordagem acerca da consulta de } \\
\text { enfermagem ginecológica }\end{array}$ & $\begin{array}{l}\text { Promoçãoda } \\
\text { educação sexual } \\
\text { da mulher }\end{array}$ & Hipermídia \\
\hline $\mathrm{E} 4^{15}$ & $\begin{array}{l}2012 \\
\text { Freitas LV, } \\
\text { et al. }\end{array}$ & $\begin{array}{c}\text { Exame físico no pré-natal: construção e } \\
\text { validação de hipermídia educativa para a } \\
\text { Enfermagem }\end{array}$ & $\begin{array}{l}\text { Promoção da } \\
\text { saúde da gestante }\end{array}$ & Hipermídia \\
\hline $\mathrm{E} 5^{16}$ & $\begin{array}{l}2012 \\
\text { Oliveira } \\
\text { MG. }\end{array}$ & $\begin{array}{c}\text { Manual saúde sexual e reprodutiva: } \\
\text { métodos anticoncepcionais } \\
\text { comportamentais - desenvolvimento e } \\
\text { avaliação de tecnologia assistiva }\end{array}$ & $\begin{array}{l}\text { Promoçãoda } \\
\text { educação sexual } \\
\text { da mulher }\end{array}$ & Manual \\
\hline $\mathrm{E} 6^{17}$ & $\begin{array}{l}2012 \\
\text { Reberte LM, } \\
\text { Hoga LAK, } \\
\text { Gomes ALZ. }\end{array}$ & $\begin{array}{c}\text { O processo de construção de material } \\
\text { educativo para a promoção da saúde da } \\
\text { gestante }\end{array}$ & $\begin{array}{l}\text { Promoção da } \\
\text { saúde da gestante }\end{array}$ & Cartilha \\
\hline$E 7^{18}$ & $\begin{array}{c}2013 \\
\text { Moraes JL. }\end{array}$ & $\begin{array}{l}\text { Protocolo para consulta de enfermagem no } \\
\text { prénatal: construção e validação }\end{array}$ & $\begin{array}{l}\text { Promoção da } \\
\text { saúde da gestante }\end{array}$ & Protocolo \\
\hline $\mathrm{E}^{19}$ & $\begin{array}{l}2015 \\
\text { Cavalcante } \\
\text { LDW, et al. }\end{array}$ & $\begin{array}{l}\text { Tecnologia assistiva para mulheres com } \\
\text { deficiência visual acerca do preservativo } \\
\text { feminino: estudo de validação }\end{array}$ & $\begin{array}{l}\text { Promoçãoda } \\
\text { educação sexual } \\
\text { da mulher }\end{array}$ & $\begin{array}{l}\text { Hipermídia e } \\
\text { simuladores }\end{array}$ \\
\hline $\mathrm{E} 9^{20}$ & $\begin{array}{l}2016 \\
\text { Peuker AC, } \\
\text { et al. }\end{array}$ & $\begin{array}{l}\text { Construção de um material educativo para } \\
\text { a prevenção do câncer de colo do útero }\end{array}$ & $\begin{array}{l}\text { Prevenção de } \\
\text { doenças }\end{array}$ & Cartilha \\
\hline $\mathrm{E} 10^{21}$ & $\begin{array}{l}2016 \\
\text { Melo GP, et } \\
\text { al. }\end{array}$ & $\begin{array}{l}\text { Elaboração e validação do protocolo } \\
\text { assistencial de enfermagem para sala de } \\
\text { pré-parto, parto e pós-parto }\end{array}$ & $\begin{array}{l}\text { Promoção do } \\
\text { conforto da } \\
\text { parturiente }\end{array}$ & Protocolo \\
\hline $\mathrm{E} 11^{22}$ & $\begin{array}{l}2016 \\
\text { Albuquerque } \\
\text { AFLL, et al. }\end{array}$ & $\begin{array}{c}\text { Tecnologia para o autocuidado da saúde } \\
\text { sexual e reprodutiva de mulheres } \\
\text { estomizadas }\end{array}$ & $\begin{array}{l}\text { Promoçãoda } \\
\text { educação sexual } \\
\text { da mulher }\end{array}$ & Cartilha \\
\hline $\mathrm{E} 12^{23}$ & $\begin{array}{l}2016 \\
\text { Abissulo CMF, } \\
\text { Silvino ZR, } \\
\text { Ferreira HC. }\end{array}$ & $\begin{array}{c}\text { Validação de simuladores realísticos para } \\
\text { orientação sobre aleitamento materno: } \\
\text { pesquisa quase experimental }\end{array}$ & $\begin{array}{l}\text { Promoção do } \\
\text { aleitamento } \\
\text { materno }\end{array}$ & Simuladores \\
\hline $\mathrm{E} 13^{24}$ & $\begin{array}{l}2017 \\
\text { Souza AA, et al. }\end{array}$ & $\begin{array}{l}\text { Construção e validação de cartilha } \\
\text { educativa sobre endometriose }\end{array}$ & $\begin{array}{l}\text { Reabilitação da } \\
\text { saúde da mulher }\end{array}$ & Cartilha \\
\hline $\mathrm{E} 14^{25}$ & $\begin{array}{c}2017 \\
\text { Primo CC, } \\
\text { Brandão MAG. }\end{array}$ & $\begin{array}{c}\text { Teoria Interativa de Amamentação: } \\
\text { elaboração e aplicação de uma teoria de } \\
\text { médio alcance }\end{array}$ & $\begin{array}{l}\text { Promoção do } \\
\text { aleitamento } \\
\text { materno }\end{array}$ & Teoria \\
\hline
\end{tabular}




\begin{tabular}{|c|c|c|c|c|}
\hline No & Ano/Autor & Título & Temática & $\begin{array}{l}\text { Tecnologia } \\
\text { utilizada }\end{array}$ \\
\hline $\mathrm{E} 15^{26}$ & $\begin{array}{c}2018 \\
\text { ResendeFZ. }\end{array}$ & $\begin{array}{c}\text { Aplicativo educacional para apoiar o } \\
\text { ensino do processo de enfermagem na } \\
\text { assistência à mulher, à criança e à família } \\
\text { em amamentação }\end{array}$ & $\begin{array}{l}\text { Promoção do } \\
\text { aleitamento } \\
\text { materno }\end{array}$ & Aplicativo \\
\hline $\mathrm{E} 16^{27}$ & $\begin{array}{c}2018 \\
\text { Bezerra RA. }\end{array}$ & $\begin{array}{l}\text { Cuidado de enfermagem à saúde da } \\
\text { gestante: aplicativo móvel para o } \\
\text { autocuidado no controle da pressão arterial }\end{array}$ & $\begin{array}{l}\text { Promoção da } \\
\text { saúde da gestante }\end{array}$ & Aplicativo \\
\hline $\mathrm{E} 17^{28}$ & $\begin{array}{c}2018 \\
\text { Silva ANB, } \\
\text { Machado MFAS. }\end{array}$ & $\begin{array}{l}\text { Desenvolvimento de um gestograma } \\
\text { destinado às gestantes no } \\
\text { acompanhamento do pré-natal }\end{array}$ & $\begin{array}{l}\text { Promoção da } \\
\text { saúde da gestante }\end{array}$ & Gestograma \\
\hline $\mathrm{E} 18^{29}$ & $\begin{array}{l}2018 \\
\text { Pereira PSL, } \\
\text { et al. }\end{array}$ & $\begin{array}{l}\text { Tecnologias não invasivas de cuidado: } \\
\text { percepção das puérperas }\end{array}$ & $\begin{array}{l}\text { Promoção do } \\
\text { conforto da } \\
\text { parturiente }\end{array}$ & $\begin{array}{c}\text { Massagem, } \\
\text { banho, } \\
\text { deambulação e } \\
\text { banqueta meia-lua }\end{array}$ \\
\hline $\mathrm{E} 19^{30}$ & $\begin{array}{l}2019 \\
\text { Cruz GCV, } \\
\text { et al. }\end{array}$ & $\begin{array}{c}\text { Construção e validação de uma tecnologia } \\
\text { educativa sobre a vacina papilomavírus } \\
\text { humano para adolescentes }\end{array}$ & $\begin{array}{l}\text { Prevenção de } \\
\text { doenças }\end{array}$ & Cartilha \\
\hline $\mathrm{E} 20^{31}$ & $\begin{array}{c}2019 \\
\text { Sousa MGP. }\end{array}$ & $\begin{array}{l}\text { Desenvolvimento e validação de um } \\
\text { protótipo de aplicativo para plataforma } \\
\text { móvel para promoção da saúde de } \\
\text { gestantes. }\end{array}$ & $\begin{array}{l}\text { Promoção da } \\
\text { saúde da gestante }\end{array}$ & Aplicativo \\
\hline $\mathrm{E} 21^{32}$ & $\begin{array}{l}2019 \\
\text { Duarte MR, } \\
\text { et al. }\end{array}$ & $\begin{array}{c}\text { Tecnologias do cuidado na enfermagem } \\
\text { obstétrica: contribuição para o parto e } \\
\text { nascimento }\end{array}$ & $\begin{array}{l}\text { Promoção do } \\
\text { conforto da } \\
\text { parturiente }\end{array}$ & $\begin{array}{l}\text { Massagem, } \\
\text { banho, bola suíça, } \\
\text { deambulação } \\
\text { aromaterapia e } \\
\text { musicoterapia }\end{array}$ \\
\hline $\mathrm{E} 22^{33}$ & $\begin{array}{l}2019 \\
\text { Franco MS, } \\
\text { et al. }\end{array}$ & $\begin{array}{c}\text { Tecnologia educacional para } \\
\text { empoderamento materno na autoeficácia } \\
\text { em amamentar }\end{array}$ & $\begin{array}{l}\text { Promoção do } \\
\text { aleitamento } \\
\text { materno }\end{array}$ & Cartilha \\
\hline $\mathrm{E} 23^{34}$ & $\begin{array}{l}2019 \\
\text { Silva RM, } \\
\text { et al. }\end{array}$ & $\begin{array}{c}\text { Uso de tecnologia móvel para o cuidado } \\
\text { gestacional: avaliação do aplicativo } \\
\text { GestAção }\end{array}$ & $\begin{array}{l}\text { Promoção da } \\
\text { saúde da gestante }\end{array}$ & Aplicativo \\
\hline
\end{tabular}

Fonte: Autoria própria.

Os estudos eleitos foram sintetizados e organizados conforme as seguintes orientações: ordem, tipo de publicação, título, autores, ano de publicação, conteúdo temático e categoria tecnológica. A partir da análise dos anos das publicações, observa-se uma acentuação intercalada com constante à produção nos 10 anos, com a seguinte distribuição: 2010 (2), 2011 (1), 2012 (3), 2013 (1), 2015 (1), 2016 (4), 2017 (2), 2018 (4), 2019 (5).

Referente ao tipo de publicação, Teses de Doutorado e Trabalhos de Conclusão de Curso ou de Especialização se mostraram ausentes na amostra final desta revisão, enquanto os demais estudos foram categorizados em: Artigo (16) e Dissertação de Mestrado (7).

No que diz respeito a classificação, a maioria dos estudos se mostraram com nível de evidência IV (21), fundamentados na categorização da $A H R Q^{11}$, uma vez que apresentam evidências oriundas de estudos descritivos, quantitativos, aplicados ou correlacionais. Já um estudo foi classificado com nível de evidência III (E12), ensaio clínico sem randomização com planejamento quase- 
experimental, e um estudo com nível de evidência VI (E14), estudos descritivos ou qualitativos. Com o objetivo de realizar a análise e discussão das tecnologias desenvolvidas no contexto da saúde da mulher, ordenou-se os estudos eleitos em categorias por classificação tecnológica de cada pesquisa, segundo a classificação de Merhy", a saber: "Estudos com foco em Tecnologia Leve"; "Estudos com foco em Tecnologia Leve-Dura" e "Estudos com foco em Tecnologia Dura" (Tabela 2).

Tabela 2 - Classificação dos estudos segundo categoria e abordagem tecnológica. Brasil, 2020.

\begin{tabular}{lll}
\hline Classificação Tecnológica & $\begin{array}{l}\text { Abordagem da } \\
\text { Tecnologia }\end{array}$ & Estudos Selecionados \\
\hline $\begin{array}{l}\text { Estudos com foco em Tecnologia } \\
\text { Leve }\end{array}$ & $\begin{array}{l}\text { Assistência } \\
\text { Educação em Saúde }\end{array}$ & E1. \\
Estudos com foco em Tecnologia E21. \\
$\begin{array}{ll}\text { Leve-Dura } \\
\text { Assistência }\end{array}$ & E7, E10, E17. \\
Estudos com foco em Tecnologia \\
$\begin{array}{ll}\text { Eura } \\
\text { Educação em Saúde em Saúde }\end{array}$ & E5, E6, E9, E11, E12, E13, E14, E19, E22. \\
\hline
\end{tabular}

Fonte: Autoria própria.

\section{Discussão}

\section{Estudos com foco em Tecnologia Leve}

Na categoria "Estudos com foco em Tecnologia Leve" foram eleitos cinco pesquisas que contemplaram a abordagem da tecnologia na assistência e na educação em saúde. A grande maioria dos estudos buscou conhecer, por intermédio da percepção da puérpera, as tecnologias não invasivas de cuidado durante o parto realizadas por enfermeiras, cujo objetivo era o alívio da dor e ansiedade na parturição ${ }^{13,29,32}$.

As puérperas que usufruíram das tecnologias de cuidado para a atenuação da dor no processo de parto as julgaram como ótimo e de grande importância, uma vez que proporcionaram bem-estar e diminuição do tempo de espera durante o trabalho de parto ${ }^{29}$. As puérperas identificaram a ação aprazível e prática da livre movimentação corporal, o incentivo à presença do acompanhante, banho de aspersão, massagem, uso da bola suíça, aromaterapia e musicoterapia, como as principais tecnologias não farmacológicas executadas pelas enfermeiras obstetras ${ }^{13,32}$.

O estudo de Nunes propôs a utilização de atividades educativas em saúde como tecnologias positivas na identificação de problemas da comunidade assistida pela Estratégia Saúde da Família (ESF). Segundo o autor, a usabilidade de tecnologias educativas na promoção de saúde possibilitou mudanças comportamentais de um grupo de mulheres adultas jovens, incluindo este grupo de usuárias na aprendizagem e na troca de saberes científicos e populares, o que corroborou na discussão dos problemas por elas enfrentadas, assim como o desenvolvimento de soluções práticas, eficazes e de baixo custo ${ }^{12}$.

Nesse contexto, percebe-se pela relação temporal dos estudos a evolução usual das tecnologias leves nos ciclos de vida da mulher, mais precisamente nos períodos gravídico e puerperal, seja 
na promoção de saúde ou na assistência a esse público. Todos os estudos demonstraram que, a postura e o uso ético e seguro pela enfermagem de tecnologias leves, colaboram para uma adequada adesão e assimilação das mulheres sobre sua relevância na resolução dos problemas que possam se apresentar nos ciclos de sua vida, tornando-as mais autônomos e protagonistas desse processo.

\section{Estudos com foco em Tecnologia Leve-Dura}

$\mathrm{Na}$ categoria "Estudos com foco em Tecnologia Leve-Dura" selecionou-se 12 estudos que contemplavam a construção e validação ou avaliação de cartilhas, ferramentas, manuais, protocolos e teorias na assistência e na educação em saúde $16-18,20-25,30,33$. A maioria dos estudos desenvolveram cartilhas educativas para promoção do autocuidado e prevenção de agravos à saúde da mulher nas fases reprodutiva, gestacional e puerperal, uma vez que abordaram as seguintes temáticas: saúde da gestante, saúde sexual e reprodutiva de mulheres estomizadas, câncer do colo de útero, endometriose, vacina contra papilomavírus humano e amamentação no período pós-parto 17,20,22,24,30,33. Todos os estudos traziam a relevância do uso das tecnologias criadas para a aquisição de conhecimento, que, por sua vez, subsidiara o empoderamento no autocuidado do público alvo ${ }^{17,20-22,24,30,33}$.

Dois estudos desenvolveram ferramentas/instrumentos de baixa e alta fidelidade enquanto tecnologias inovadoras para educação e orientação. O estudo de Abissulo propôs o uso de simuladores realísticos, confeccionados artesanalmente, de baixo custo e manutenção, para orientar as puérperas sobre aleitamento materno. Sua utilização alterou o conhecimento e o desempenho das puérperas sobre o manejo do aleitamento materno, suprimindo as dificuldades evidenciadas por elas durante esse processo ${ }^{23}$. Já o estudo de Silva e Machado apresentou um novo tipo de gestograma como tecnologia de promoção da saúde, com a finalidade permitir à gestante o acompanhamento de sua gestação por meio das informações impressas no instrumento. Os autores buscaram criar e/ou aprimorar instrumentos que permitissem suprir dúvidas das usuárias acerca de saúde ${ }^{28}$.

Ainda com foco nas tecnologias leve-dura, dois estudos apresentaram seus protocolos assistenciais direcionados a consulta de enfermagem no pré-natale aos cuidados de enfermagem na sala de pré-parto, parto e puerpério (PPP). Consideraram-se protocolos estruturados com qualidade técnica e científica, sendo validados em conteúdo e aparência por especialistas, indicando-se, portanto, suas utilizações na prática de enfermagem na ESF e nas maternidades ${ }^{18}$, 21.

Outro estudo selecionado tinha como cerne o desenvolvimento e a avaliação de uma tecnologia assistiva, cujo prisma era os métodos contraceptivos comportamentais para mulheres cegas ${ }^{16}$. Tecnologia assistiva, segundo Bersch e Pelosi, é o termo usado para nomear o grupo de aparatose técnicas que promovam ou amplifiquem aptidões funcionais de Pessoas com Deficiência (PcD) e idosos, proporcionando a maximização da independência, qualidade de vida e inclusão social ${ }^{36}$. O manual apresenta texto em Braile e em tinta, com figuras em alto relevo acompanhados de descrição simples, clara e objetiva ${ }^{16}$.

O estudo de Primo e Brandão ${ }^{25}$ descreve a criação de uma teoria de médio alcance fundamentada no Modelo Conceitual de Sistemas Abertos de Imogene King e os princípios do método de amamentar. Por apresentar fundamento em um modelo conceitual da enfermagem e apresentar uma característica de abstração, essa tecnologia pode ser implementada nos diferentes 
contextos da comunidade, uma vez que conceitua a amamentação de forma ampla, dinâmica e processual, além de contribuir para a ciência da enfermagem.

Vale ressaltar que a maioria dos estudos passou por diferentes fases de desenvolvimento até serem validados ou avaliados por juízes especialistas da área de enfermagem, de outras ciências, e de usuários. Usando essas tecnologias, a enfermagem, até mesmo outros profissionais, poderão oferecer suporte educacional e técnico no manejo e conduta de uma gama de situações que envolvem a saúde da mulher.

\section{Estudos com foco em Tecnologia Dura}

Nesta categoria, sete estudos foram eleitos conforme seu cerne no desenvolvimento e/ ou validação de aplicativos móveis. Nessa categoria, três APP têm como objetivo auxiliar as grávidas interessadas em conseguir informações sobre a fase gravídica e o autocuidado no controle da pressão arterial ${ }^{27-28,31}$. Outros três estudos desenvolveram hipermídias que têm como finalidade apresentar o processo de criação e validação de tecnologias para o apoio do processo ensino-aprendizagem da consulta e da assistência de enfermagem em ginecologia, pré-natal e amamentação ${ }^{14-15,26 .}$

Um estudo visou validar uma tecnologia assistiva denominada "Construir para aprender a usar o preservativo feminino" para mulheres com deficiência visual. A TA apresentou em todos os itens avaliados pelos juízes especialistas um nível de concordância de $70 \%$. Dessa forma, foi validada e classificada como adequada, ou seja, é uma ferramenta inovadora e de baixo custo que pode auxiliar mulheres deficientes visuais quanto ao manuseio do preservativo feminino ${ }^{19}$.

As informações examinadas nesta revisão mostraram que os estudos na vertente de pesquisa de tecnologias inovadoras aplicadas no contexto da saúde da mulher no Brasil é uma área nova e em progressiva ascensão. Os estudos identificados entre os anos de 2009 e 2019 apresentaram maior centralização no ano de 2019

Os estudos na vertente de pesquisa de tecnologias inovadoras aplicadas no contexto da saúde da mulher no Brasil é uma área nova e em progressiva ascensão. com cinco estudos. Se observou uma ampliação das publicações significativas no que se refere ao objeto de pesquisa ao longo dos anos estudados e julga-se que esse número será ainda mais significativo nos próximos anos, uma consequência da popularização da internet e das tecnologias móveis.

A análise das publicações evidenciou que a categoria tecnológica mais focada no desenvolvimento de instrumentos assistências, educacionais e de ensino foi a tecnologia levedura com doze estudos, que segundo Merhy, integra o conjunto e conhecimentos estruturados e retratados pelos princípios que são empregados na saúde. As temáticas que foram tratadas exclusivamente dizem respeito a assistência e a educação ${ }^{4}$.

No meio dos estudos examinados fora possível reconhecer três aplicativos que focam como público final as mulheres e os profissionais de enfermagem. A construção de APP, seja para dar apoio a mulher ou ao profissional enfermeiro, é passível de ser evidenciada como um espaço a ser melhor investigado. Uma das qualidades marcantes dos celulares inteligentes é a usabilidade quanto a resolução de tarefas, seja qual for a fase do dia, uma vez que esta tecnologia se faz acessível 24 horas, podendo ser transportada e manuseada em qualquer lugar. No que diz respeito a ciência enfermagem, esta característica dos dispositivos móveis pode representar 
um relevante auxílio na disseminação de informações e qualificação das boas práticas nos contextos do ensino, da pesquisa e da assistência.

\section{Conclusões}

A maior parte dos estudos eleitos tinha como foco o desenvolvimento de tecnologia leve e leve-dura com abordagem na educação em saúde desse público, o que destaca o esforço dos profissionais em tornar a mulher protagonista de todas as fases de sua vida.

Ainda que exista discreto quantitativo de estudos desenvolvidos no Brasil sobre essa temática, nota-se uma evolução contínuo ao longo da década analisada. Todavia, nota-se que os pesquisadores e as tecnologias duras concentram a maior parte de seus objetivos na fase reprodutiva e gestacional da mulher, não observando que o cenário de morbimortalidade desse público, seja a nível mundial ou nacional, perpassa por complicações evitáveis relacionadas à gestação, ao parto e ao puerpério.

O levantamento dos estudos evidenciou que existe uma ascensão de pesquisas com foco na promoção, prevenção e reabilitação da mulher por intermédio das mais diversas tecnologias em saúde, seja do acolhimento a esse público ao mundo virtual da web. No entanto, devese destacar que qualquer modalidade tecnológica deve passar por análise e testagem de profissionais qualificados e que conheçam a real necessidade do público alvo, uma vez que o produto final será coerente a demanda pela qual foi criado, testado na pesquisa e implementado na prática.

Para as pesquisas futuras, sugere-se que, além das tecnologias identificadas, torna-se necessário explorar ainda mais as tecnologias classificadas como duras, especificamente as tecnologias informatizadas em dispositivos móveis, a julgar pelo atual cenário da era digital em que a adoção de tecnologias para o cuidado proporciona maior resolubilidade, agilidade e segurança na avaliação do cliente, além da coleta, manuseio e armazenamento de dados.

Conflitos de interesse: Os autores declaram que não houve conflitos de interesse.

Financiamento: Fundação de Amparo à Pesquisa do Estado do Amazonas.

\section{Referências}

1. Shoji S, Souza NVDO, Maurício VC, Costa CCP, Alves FT. O cuidado de enfermagem em estomaterapia e o uso das tecnologias. Estima. 2017; 15(3): 169-77.

http://dx.doi.org/10.5327/Z1806-3144201700030008

2. Engela MHT, Rodarte AC, Rotondaro Júnior A, Seixas CT, Viegas SMF, Lanza FM. Uso das tecnologias em saúde na atenção básica às pessoas em condições de hipertensão arterial sistêmica. Rev Fund Care Online. 2018; 10(1): 75-84.

http://dx.doi.org/10.9789/2175-5361.2018.v10i1.75-84

3. Lorenzetti J, Trindade LL, Pires DEP, Ramos FRS. Tecnologia, inovação tecnológica e saúde: uma reflexão necessária. Texto \& Contexto Enfermagem. 2012; 21(2): 432-39.

http://dx.doi.org/10.1590/S0104-07072012000200023

4. Merhy EE. Saúde: a cartografia do trabalho vivo. 2. ed. São Paulo: Hucitec; 2005. 
5. Organização Pan-Americana da Saúde. OPAS. Folha informativa - Mortalidade materna [internet]. 2018. [cited 27 Jun 2019]; Available from: https://www.pa-ho.org/bra/index. php?option=com_content\&view=article\&id=5741:folha-informativa-mortalidade-materna\&Itemid $=820$

6. World Health Organization. MHealth: new horizons for health through mobile technologies: based on the findings of the second global survey on ehealth. Geneva: World Health Organization; 2011.

7. Silva AMA, Mascarenhas VHA, Araújo SNM, Machado RS, Santos AMR, Andrade EMLR. Mobile technologies in the Nursing tech. Rev Bras Enferm. 2018; 71(5): 2570-8. http://dx.doi.org/10.1590/0034-7167-2017-0513

8. Polit DF, Beck CT. Fundamentos de Pesquisa em enfermagem: avaliação de evidências para as práticas da enfermagem. 9. ed. Porto Alegre: Artmed; 2019.

9. Brasil. Ministério da Saúde. Diretrizes e normas regulamentadoras de pesquisa envolvendo seres humanos. Brasília: Ministério da Saúde; 2012.

10.Souza MT, Silva MD, Carvalho R. Revisão integrativa: o que é e como fazer. Einstein. 2010, 8(1):102-106. http://dx.doi.org/10.1590/s1679-45082010rw1134

11.Galvão CM. Níveis de evidência. Acta Paul. Enferm. 2006; 19(02). http://dx.doi.org/10.1590/S0103-21002006000200001

12.Nunes JM. Tecnologia educativa: uma proposta para promoção da saúde de um grupo de mulheres. [dissertação] [Internet]. Fortaleza: Universidade Federal do Ceará; 2010. [cited 05 mar 2020]. Available from:

http://www.repositorio.ufc.br/bitstream/riu-fc/1800/1/2010_dis_jmnunes.pdf

13. Nascimento NMD, Progianti JM, Novoa RI, Oliveira TRD, Vargens OMDC. Tecnologias não invasivas de cuidado no parto realizadas por enfermeiras: a percepção de mulheres. Escola Anna Nery. 2010; 14(3): 456-61. http://dx.doi.org/10.1590/S1414-81452010000300004

14. Morais MLCD. Construção e validação de hipermídia educacional em saúde sexual: uma abordagem acerca da consulta de enfermagem ginecológica. [dissertação] [Internet]. Fortaleza: Universidade Federal do Ceará; 2011. [cited 05 mar 2020]. Available from: http://www.repositorio.ufc.br/bitstream/riufc/2734/1/2011_dis_mlcmorais.pdf

15.Freitas LV, Teles LMR, Lima TM, Vieira NFC, Barbosa RCM, Pinheiro AKB, et al. Exame físico no pré-natal: construção e validação de hipermídia educativa para a Enfermagem. Acta Paulista de Enfermagem. 2012; 25(4): 581-588. https://doi.org/10.1590/S0103-21002012000400016

16.Oliveira MGD. Manual saúde sexual e reprodutiva: métodos anticoncepcionais comportamentais-desenvolvimento e avaliação de tecnologia assistiva. [dissertação] [Internet]. Fortaleza: Universidade Federal do Ceará; 2012. [cited 05 mar 2020]. Available from:

http://www.repositorio.ufc.br/bitstream/riufc/6990/1/2012_dis_mgoliveira.pdf

17.Reberte LM, HOGA LAK, GOMES ALZ. O processo de construção de material educativo para a promoção da saúde da gestante. Rev. Latino-Am. Enfermagem. 2012; 20(1): 101-08. https://doi.org/10.1590/S0104-11692012000100014

18. Moraes JLD. Protocolo para consulta de enfermagem no pré-natal: construção e validação. [dissertação] [Internet]. Fortaleza: Universidade Federal do Ceará; 2013. [cited 05 mar 2020]. Available from: http://www.repositorio.ufc.br/bitstream/riufc/8492/1/2013_dis_jl-moraes.pdf

19.Cavalcante LDW, Oliveira GOB, de Almeida PC, de Almeida Rebouças CB, Pagliuca LMF. Tecnologia assistiva para mulheres com deficiência visual acerca do preservativo feminino: estudo de validação. Revista da Escola de Enfermagem da USP. 2015; 49(1): 14-21.

https://doi.org/10.1590/S0080-623420150000100002

20.Peuker, AC, Lima NBD, Freire KDM, Oliveira CMMD, Castro EKD. Construção de um material educativo para a prevenção do câncer de colo do útero. Estudos Interdisciplinares em Psicologia. 2017; 8(2): 146-60. http://dx.doi.org/10.5433/2236-6407.2017v8n2p146 
21.Melo GP, Andreto LM, Araújo, VMG, Holanda VR. Elaboração e validação do protocolo assistencial de enfermagem para sala de pré-parto, parto e pós-parto. Revista Eletrônica de Enfermagem. 2016; 18. https://doi.org/10.5216/ree.v18.40589

22.Albuquerque AFLL, Pinheiro AKB, Linhares FMP, Guedes TG. Tecnologia para o autocuidado da saúde sexual e reprodutiva de mulheres estomizadas. Revista Brasileira de Enfermagem. 2016; 69(6): 1164-71. https://doi.org/10.1590/0034-7167-2016-0302

23.Abissulo CMF, Silvino ZR, Ferreira HC. Validação de simuladores realísticos para orientação sobre aleitamento materno: pesquisa quase-experimental. Online Braz. J. Nurs. [Internet]. 2016 [cited 05 mar 2020]; 15(4): 599-604. https://doi.org/10.17665/1676-4285.20165345

24.Souza AA, Serafim AIS, Sousa FAD, Souza GKT, Silva ISR, Lima LR. Construção e validação de cartilha educativa sobre endometriose. Mostra Interdisciplinar do curso de Enfermagem. 2019; 3(1).

25.Primo CC, Brandão MAG. Teoria Interativa de Amamentação: elaboração e aplicação de uma teoria de médio alcance. Revista Brasileira de Enfermagem. 2017; 70(6), 1191-98.

https://doi.org/10.1590/0034-7167-2016-0523

26. Resende FZ. Aplicativo educacional para apoiar o ensino do processo de enfermagem na assistência à mulher, à criança e à família em amamentação. [dissertação] [Internet]. Espírito Santo: Universidade Federal do Espírito Santo; 2018. [cited 05 mar 2020]. Available from: http://repositorio.ufes.br/bitstream/10/8323/1/tese_12353_disserta\%c-3\%a7\%c3\%a3o\%20 revisada_final\%20Fabiola.pdf

27. Bezerra RA. Cuidado de enfermagem à saúde da gestante: aplicativo móvel para autocuidado no controle da pressão arterial. [dissertação] [Internet]. Redenção: Universidade da Integração Internacional da Lusofonia Afro-brasileira; 2018. [cited 05 mar 2020]. Available from: http://www.repositorio.unilab.edu.br:8080/xmlui/bitstream-/handle/123456789/884/ Disserta\%c3\%a7\%c3\%a3o\%20Raylla\%20finalizada.pdf?sequence=1\&isAllowed=y

28.Silva ANB, Machado MFAS. Desenvolvimento de um Gestograma destinado às gestantes no acompanhamento do pré-natal. In: Moreira TMM, Pinheiro JAM, Florêncio RS, Cestari VRF. Tecnologias para a promoção e o cuidado em saúde. Fortaleza: EdUECE; 2018. p. 291310.

29.Pereira PSL, Gomes IS, Ribeiro ÍAP, Morais JDC, Gouveia MTDO, Nascimento MVF, et al. Tecnologias não invasivas de cuidado: percepção das puérperas. Rev. Enferm. UFPE. 2018; 12(8): 2129-36. http://dx.doi.gov/10.5205/1981-v12i8a236584p2129-2136-2018

30.Cruz GDCV, Vasconcelos MGF, Maniva SJCDF, Carvalho REFLD. Construção e validação de uma tecnologia educativa sobre a vacina papilomavírus humano para adolescentes. Escola Anna Nery. 2019; 23(3). https://doi.org/10.1590/2177-9465-ean-2019-0050

31.Sousa MGPD. Desenvolvimento e validação de um protótipo de aplicativo para plataforma móvel para promoção da saúde de gestantes. [dissertação] [Internet]. Fortaleza: Universidade Federal do Ceará; 2019. [cited 05 mar 2020]. Available from:

http://www.repositorio.ufc.br/bitstream/riufc/48769/1/2019_dis_mgpsousa.pdf

32.Duarte MR, Alves VH, Rodrigues DP, de Souza KV, Pereira AV, Pimentel MM. Tecnologias do cuidado na enfermagem obstétrica: Contribuição para o parto e nascimento. Cogitare Enfermagem. 2019; 24. http://dx.doi.org/10.5380/ce.v24i0.54164

33.Franco M.S, Carvalho JW, Lira DS, Reis ER, Cirino IP, Lima LHO. Educational technology for empowerment in maternal breastfeeding self-efficacy. Revista de Enfermagem UFPE. 2019; 13. https://doi.org/10.5205/1981-8963.2019.240857

34.Silva RM, Brasil CCP, Bezerra IC, Queiroz FFSN. Mobile health technology for gestational care: evaluation of the GestAção's App. Rev Bras Enferm. 2019; 72(Suppl 3): 266-73.

http://dx.doi.org/10.1590/0034-7167-2018-0641

35. Bersch RCR, Pelosi MB. Portal de ajudas técnicas para a educação: equipamento e material pedagógico para a educação, capacitação e recreação da pessoa com deficiência física: tecnologia assistiva: recursos de acessibilidade ao computador. Brasília: MEC, Secretaria de Educação Especial; 2007.Agnimporem harupta ssinus simus eum que net estibus re etur, imil 